\title{
PROBLEME DE LA DIFFUSION GAZEUSE DANS L'OREILLE MOYENNE AU COURS DE L'ANESTHESIE POUR TYMPANOPLASTIE
}

\author{
Luc Perreault, Pierre Rousseau, Jean-François Garneau et Guy Forget
}

\begin{abstract}
RÉSUMÉ
Les problèmes de diffusion gazeuse dans l'oreille moyenne au cours de l'anesthésie ont été décrits depuis 1965. L'administration de protoxyde d'azote provoque des élévations de pression de l'ordre de $3.43 \mathrm{kPa}$ à $3.92 \mathrm{kPa}\left(350\right.$ à $\left.400 \mathrm{mmH}_{2} \mathrm{O}\right)$ à l'intérieur de l'oreille. Ces phénomènes disparaissent spontanément lorsque cesse l'administration du protoxyde d'azote et ne laissent pas de séquelle sauf dans certains cas où on a noté de la surdité et même des ruptures tympaniques. Nous avons essayé de résoudre ce problème au cours de l'anesthésie pour greffe tympanique en substituant l'air au protoxyde d'azote et en employant l'éthranc comme agent anesthésique de base. On potentialise l'anesthésie avec des narcotiques et on maintient une curarisation adéquate. Les résultats ont montré que l'emploi de cette technique rẻduit au minimum la diffusion gazeuse si on maintient la $\mathrm{Pa}_{0_{2}}$ au-dessous de $17.9 \mathrm{kPa}$ (135 $\mathrm{mmHg}$ ) et la $\mathrm{Pa}_{\mathrm{CO}_{2}}$ au niveau de $3.9 \mathrm{kPa}(30 \mathrm{mmHg})$. Des contrôles ont montré que la diffusion est plus grande si la $\mathrm{Pa}_{0_{2}}$ est plus élevée. La pression dans l'oreille moyenne, mesurée avec l'impédence-mètre de Madsen, n'a pas dépassé $0.68 \mathrm{kPa}\left(70 \mathrm{mmH}_{2} \mathrm{O}\right)$.
\end{abstract}

Mots Clefs: Anesthesie, tympanoplastie; L'Oreille Moyen, diffusion gazeuse.

Le PHÉNOMÈNE DE LA DIFFUSION GAZEUSE danS l'oreille moyenne au cours de l'anesthésie générale a fait l'objet d'observations intéressantes par plusieurs auteurs. Dès 1965, Thomsen' et ses collaborateurs ont démontré qu'il se produit une augmentation importante de la pression intraauriculaire au cours de l'anesthésie au protoxyde d'azote. Cette augmentation de pression est de l'ordre de $3.43 \mathrm{kPa}\left(350 \mathrm{mmH}_{2} \mathrm{O}\right)$. En 1975, Patterson et Bartlett ${ }^{2}$ ont rapporté le même phénomène. Ils ont démontré qu'il se produit une élévation de pression qui atteint $3.92 \mathrm{kPa}$ $\left(400 \mathrm{mmH}_{2} \mathrm{O}\right)$ avec des chutes à $1.96 \mathrm{kPa}$ $\left(200 \mathrm{mmH}_{2} \mathrm{O}\right)$ occasionnées par l'ouverture périodique de la trompe d'Eustache. En 1967 , Matz, Rattenborg et Haladay ${ }^{3}$ ont décrit le même phénomène et ils ont étudié les modalités des échanges de l'azote et du protoxyde d'azote au cours de l'anesthésie. Ils ont fait la relation entre ce phénomène et unéélévation correspondante de la pression dans l'oreille moyenne. D'autre part, Waun, Sweitzer et Hamilton ${ }^{4}$ en 1967, ont observé qu'il peut se produire une diminution de

Luc Perreault, M.D., Pierre Rousseau, M.D., JeanFrançois Garneau, M.D., Département d'AnesthésieRéanimation; Guy Forget, M.D., Service d'Otorhino-laryngologie, Hôpital Maisonneuve-Rosemont, Montréal.

Adresse postale: Luc Perreault, M.D., Hôpital Maisonneuve-Rosemont, Département d'AnesthésieRéanimation, 5415 boul. l'Assomption, Montréal, P.Q., Canada H IT 2M4.

Canad. Anaesth. Soc. J., vol. 28, no. 2, March 1981 l'acuité auditive à la suite de l'administration du protoxyde d'azote. Enfin, Owens et ses collaborateurs ${ }^{5}$ ont même rapporté des ruptures tympaniques consécutives à l'administration de hautes concentrations du protoxyde d'azote.

Ces phénomènes de diffusion ne sont certes pas négligeables mais dans la pratique courante, tout semble rentrer dans l'ordre après quelques heures dans la presque totalité des cas. Tel n'est pas le cas cependant dans la chirurgie du tympan. En effet, l'augmentation de pression dans l'oreille moyenne après la pose d'un greffon tympanique peut amener une déhiscence de ce dernier avec persistance d'orifice mettant en communication l'oreille moyenne avec le conduit auditif externe. On peut retrouver aussi un défaut de contact avec le marteau et l'anneau tympanique ou, une greffe externe.

Nous avons donc étudié les phénomènes de diffusion dans l'oreille moyenne et nous avons essayé de réaliser une technique d'anesthésie qui perturbe au minimum l'équilibre physiologique de l'atmosphère de l'oreille moyenne.

Après avoir expérimenté des concentrations décroissantes de protoxyde d'azote nous avons réalisé que les problèmes de diffusion demeuraient entiers. En effet, l'augmentation des concentratigns d'oxygène consécutive à la diminution du protoxyde d'azote amenait des perturbations aussi importantes de l'équilibre gazeux. Nous avons donc porté notre intérêt sur 136 
un mélange plus physiologique composé d'air additionné d'une faible quantité d'oxygène. L'anesthésie était réalisée au moyen d'éthrane à des concentrations de 0.5 à 1.5 pour cent potentialisé avec fentanyl et curare.

Ce dernier mélange a l'avantage de contenir à \pm 95 pour cent des gaz que l'on rencontre dans l'oreille moyenne après la pose du greffon tympanique. En effet, la pose de ce dernier emprisonne à l'intérieur une atmosphère identique à l'atmosphère ambiante, i.e. de l'air. Dès la fermeture de la brèche tympanique, les capillaires sanguins amorcent un équilibre entre les gaz sanguins et l'atmosphère de l'oreille moyenne. Normalement, la $\mathrm{Pa}_{\mathrm{O}_{2}}$ est à $13.3 \mathrm{kPa}$ $(100 \mathrm{mmHg})$ et la $\mathrm{Pa}_{\mathrm{CO}_{2}}$ à $5.32 \mathrm{kPa}(40 \mathrm{mmHg})$. Dans l'air atmosphérique, la $\mathrm{Po}_{2}$ est à $21.14 \mathrm{kPa}$ $(159 \mathrm{mmHg})$. L'azote atmosphérique est en équilibre avec l'azote de l'organisme. Les phénomènes de diffusion se feront donc aux dépens de l'oxygène, du dioxide de carbonne et de toute autre substance gazeuse ou volatile qui se retrouvera dans le sang artériel. Dans la technique employée, seul l'éthrane vient s'ajouter aux substances diffusibles, et sa pression partielle d'environ $0.8 \mathrm{kPa}(7 \mathrm{mmHg})$ dans l'air alvéolaire est négligeable. La figure I illustre cet equilibre.

Pour éviter une augmentation du volume gazeux dans l'oreille moyenne, il faut éviter d'élever indûment la $\mathrm{Pa}_{\mathrm{O}_{2}}$ de façon à pouvoir compenser au moins partiellement la diffusion du dioxide de carbonne vers l'oreille. Selon la loi de Graham, la vitesse de diffusion d'un gaz à travers une membrane est inversement proportionnelle à la racine carrée de son poids moléculaire. ${ }^{6}$ Il est donc permis de dire que l'oxygène a une vitesse de diffusion qui équivaut à 1.2 fois celle du dioxide de carbonne. Si on abaisse la $\mathrm{Pa}_{\mathrm{CO}_{2}}$ à $3.9 \mathrm{kPa}(30 \mathrm{mmHg})$, il faudra un gradient de $3.3 \mathrm{kPa}(25 \mathrm{mmHg})$ vers l'artère pour permettre d'équilibrer les volumes gazeux au moyen de l'oxygène. Ceci nous donne donc la possibilité de maintenir une $\mathrm{Pa}_{\mathrm{O}_{2}}$ de $17.9 \mathrm{kPa}(135 \mathrm{mmHg})$ et nous aurons l'équilibre illustrié a la figure 2 .

Donc une technique d'anesthésie qui maintient une $\mathrm{Pa}_{\mathrm{O}_{2}}$ à $17.9 \mathrm{kPa}(135 \mathrm{mmHg})$ et une $\mathrm{Pa}_{\mathrm{CO}_{2}}$ à $3.9 \mathrm{kPa}(30 \mathrm{mmHg})$ devrait éliminer à peu près complètement le problème de surpression dans l'oreille moyenne si on évite d'y ajouter d'autres substances hautement diffusibles.

Notre technique d'anesthésie comporte une induction avec thiopental aux doses usuelles, suivi de succinylcholine $1 \mathrm{mg} \cdot \mathrm{kg}^{-1}$. Avant l'intubation trachéal nous évitons d'administrer une
Sang artériel

Oreille moyenne

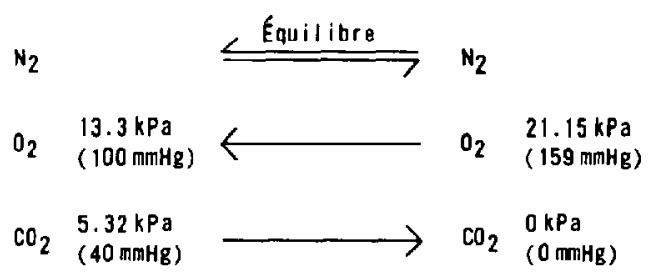

Figure 1 Etat des gaz dans le sang artériel et dans l'oreille moyenne avant la pose du tympan.

Sang arterial

Oroille moyenne

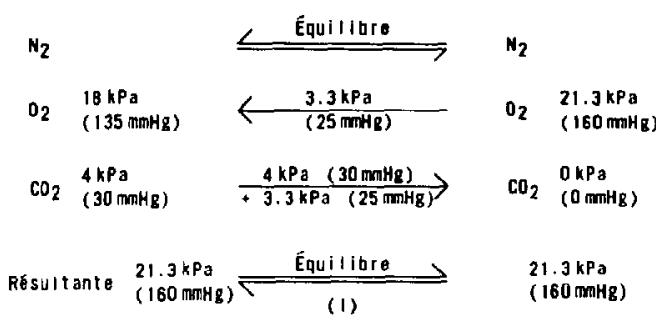

+ Corrigé pour coefficient de diffusion $\mathrm{O}_{2} / \mathrm{CO}_{2}$

(1) Total compensé par

FIGURE 2 Equilibre gazeux à rechercher après la pose du tympan.

$\mathrm{Fi}_{\mathrm{O}_{2}}$ de 1.0 pour ne pas élever la $\mathrm{Pa}_{\mathrm{O}_{2}}$ d'une façon trop marquée. Le mélange idéal est de trois $1 / \mathrm{min}$ d'air et deux $\mathrm{l} / \mathrm{min}$ de l'oxygène, ce qui donne une $\mathrm{Fi}_{\mathrm{O}}$ de 0.5. Dès que l'intubation est réalisée, nous abaissons la $\mathrm{Fi}_{\mathrm{O}_{2}}$ entre 0.25 et 0.3 et nous faisons un contrôle des gaz sanguins après 30 minutes. La ponction artérielle se fait dans l'artère pédieuse ou la tibiale postérieure avec une aiguille no. 26 pour minimiser le plus possible le traumatisme artériel. Si les chiffres de la $\mathrm{Pa}_{\mathrm{O}_{2}}$ ou de la $\mathrm{Pa}_{\mathrm{CO}_{2}}$ sont trop élevés ou trop bas, nous faisons les corrections nécessaires et nous faisons un contrôle 30 minutes plus tard lorsque la durée de l'intervention le permet.

Nous employons un circuit semi-fermé ou un circuit de Bain et la respiration est contrôlée mécaniquement. En circuit semi-fermé, le débit gazeux est maintenu à environ six litres/min et nous observons les chiffres recommandés de $70 \mathrm{ml} \cdot \mathrm{kg}^{-1} / \mathrm{min}$ lorsque nous utilisons le circuit de Bain. La $\mathrm{Fi}_{\mathrm{O}_{2}}$ est contrôlée au moyen d'un oxymètre qui mesure laqualité des gaz frais et le volume de la ventilation est réglé au départ à $100 \mathrm{ml} \cdot \mathrm{kg}^{-1} / \mathrm{min}$. Les volumes sont ajustés après la première analyse des gaz sanguins. 
Évaluation du degré de oÉplacement DE LA GREFFE TYMPANIQUE

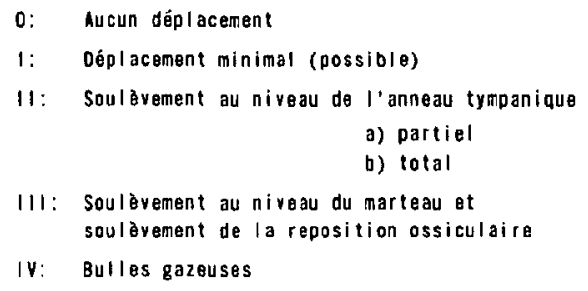

FIgure 3 Critères d’évaluation de la stabilité du greffon tympanique.

\section{ÉVALUATION DES PERTES SANGUINES}

0: Aucune

1: Suintement

11: Saignement

\section{III: Hémorragie}

Figure 4 Critère deévaluation du saignement capillaire.

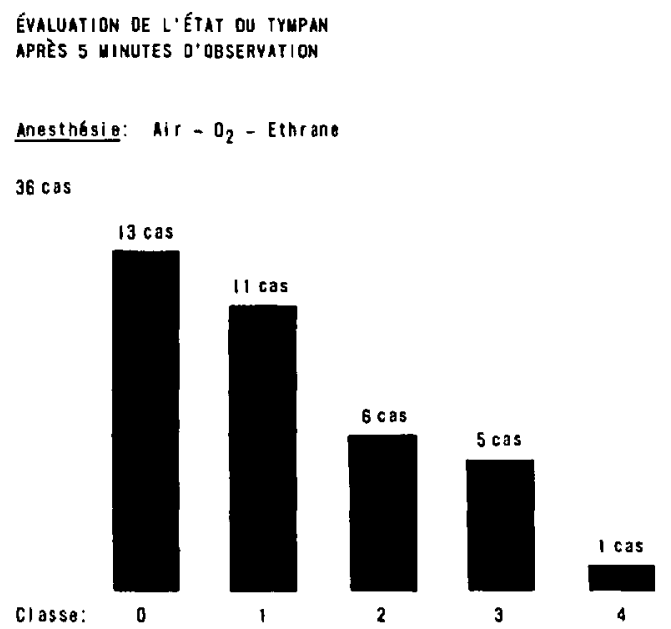

Figure 5 Résultats de l'évaluation de la stabilité tympanique.

Le fentanyl et le dropéridol sont administrés selon les besoins et nous curarisons les malades avec d-tubocurarine $0.25 \mathrm{mg}$ à $0.4 \mathrm{mg} \cdot \mathrm{kg}^{-1}$. L'étrane est maintenu à une concentration de 1.5 pour cent à l'induction et 1.0 pour cent durant le maintien.

Cette technique procure une anesthésie am-

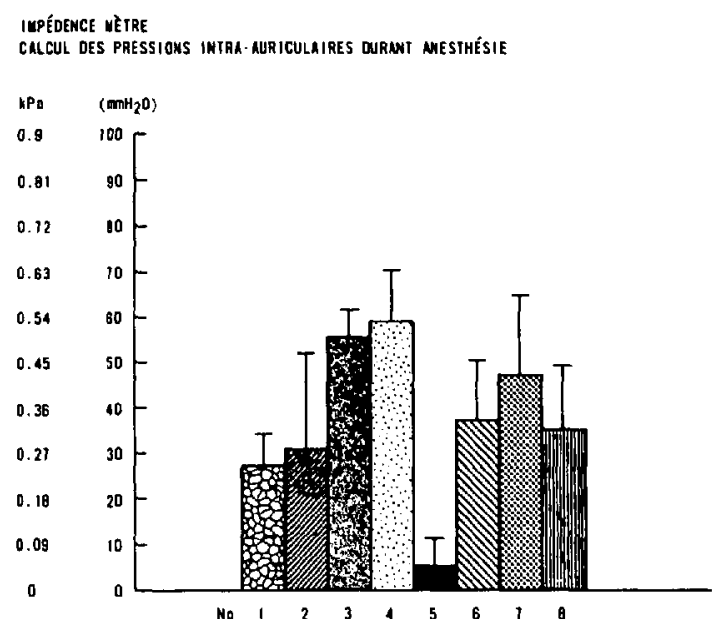

Figure 6 Pressions intra-auriculaires moyennes chez huit malades.

plement suffisante pour l'intervention. La tension artérielle subit une légère diminution et se stabilise à un niveau de 10 à 15 pour cent inférieur aux chiffres de départ. Il en est de même pour le pouls qui est remarquablement stable comme d'ailleurs au cours de toutes les anesthésies à l'éthrane. L'éveil est très rapide et l'incidence des nausées et des vomissements n'est pas augmentée. L'amnésie est complète.

La stabilité du greffon tympanique est évaluée par le chirurgien après une période d'attente de cinq minutes et les résultats sont classifiés selon la gradation exprimée à la figure 3 .

De même, le saignement capillaire subit une évaluation selon la gradation exprimée à la figure 4.

Au moyen de l'impédence-mètre de Madsen, nous avons mesuré, chez dix malades, les variations de la pression intra-auriculaire dans l'oreille opposée au site opératoire. L'appareil était installé après l'induction et l'enregistrement des pressions se faisait aux cinq minutes.

\section{Resultats}

Dans un groupe de trente-six malades, la stabilité tympanique se situe dans les catégories 0 et 1 , ce qui constitue un excellent résultat (Figure $5)$.

La répartition des résultats par rapport à la $\mathrm{Pa}_{\mathrm{O}_{2}}$ montre que la plus grande partie des bons résultats se retrouve chez les malades dont la $\mathrm{Pa}_{\mathrm{O}_{2}}$ se situe entre $10.6 \mathrm{kPa}(80 \mathrm{mmHg})$ et $18.6 \mathrm{kPa}(140 \mathrm{mmHg}$ ) (Tableau I).

La mesure de la pression intra-auriculaire ap- 
TABLEAU I

Evaluation de la Stabilité de la Membrane Tympanique en Fonction de la PaO, (Anesthésie: Air-Oxygen-Ethrane)

\begin{tabular}{llllll}
\hline $\mathrm{Pa}_{2} \quad \mathrm{kPa}(\mathrm{mmHg})$ & 0 & 1 & 2 & 3 & 4 \\
\hline 26.60 à $23.94(200$ à 180$)$ & & 1 & & 1 & \\
23.94 à $21.28(180$ à 160$)$ & 1 & 1 & 2 & & \\
21.28 à $18.62(160$ à 140$)$ & 1 & & & & \\
18.62 à $15.96(140$ à 120$)$ & 5 & 2 & 1 & 2 & \\
15.96 à $13.30(120$ à 100$)$ & 4 & 3 & 2 & 2 & \\
13.30 à $10.64(100$ à 80$)$ & 3 & 3 & 2 & & 4 \\
& Grade: 0 & 1 & 2 & 3 & 4
\end{tabular}

Les chiffres dans chacun des carrés signifient le nombre de cas.

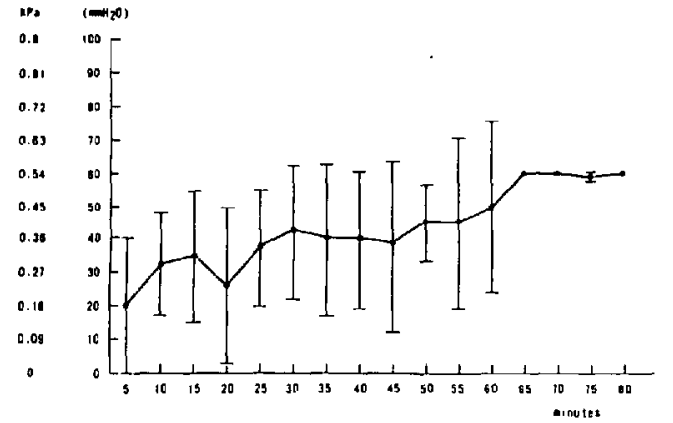

Figure 7 Pressions intra-auriculaires moyennes en fonction de la durée de l'intervention.

Évaluation DEs PERTES Sanguines

Anesthésle: Air - $0_{2}$ - Ethrane

36 cas

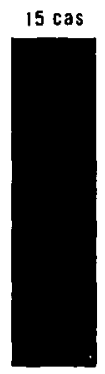

Classe:

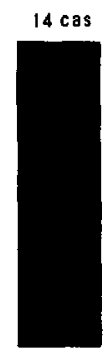

1

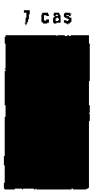

La figure 7 montre la moyenne des pressions obtenues en fonction du temps opératoire et on note une tendance à la stabilisation autour de $0.6 \mathrm{kPa}\left(60 \mathrm{mmH}_{2} \mathrm{O}\right)$ après une heure d'anesthésie.

L'évaluation du saignement capillaire montre que vingt-neuf cas sur trente-six se retrouvent dans les deux premières catégories (Figure 8).

\section{CONCLUSION}

En modifiant les agents d'inhalation, nous avons réussi à mettre au point une technique d'anesthésie qui prévient les problèmes de diffusion gazeuse au niveau de l'oreille moyenne. Cette anesthésie empêche les déhiscences de la greffe tympanique et devrait améliorer les résultats à court et à long terme dans ce genre d'anesthésie.

\section{BIBLIOGRAPHIE}

1. Thomsen, K.A., Terkildsen, K. \& Arnfred, 1. Middle ear pressure variations during anesthesia. Arch. Otolaryng. 82: 609-611,1965.

2. Patterson, Marck E. \& Bartlett, Philip C. Hearing impairment caused by intratympanic pressure changes during general anesthesia. The Laryngoscope 86: 399-404, 1976.

3. Matz, Gregory J., Rattenborg, Christen G. \& Holaday, DUNCAN A. Effects of nitrous oxide on middle ear pressure. Anesthesiology 28: 948-950, 1967.

Figure 8 Résultats de l'évaluation du saignement capillaire.

paraît à la figure 6 qui illustre les pressions moyennes de huit malades, et démontre que les pressions n'ont pas dépassé $0.68 \mathrm{kPa}$ $\left(70 \mathrm{mmH}_{2} \mathrm{O}\right)$ et que la moyenne pour tous les cas est inférieure à ce chiffre (Figure 6).
4. Waun, James E., Sweitzer, Richard S. \& Hamilton, William K. Effects of nitrous oxide on middle ear mechanics and hearing acuity. Anesthesiology 28: 846-850, 1967.

5. OWens, William D., Gustave, Frederic \& SClarofF, AlaN. Tympanic membrane rupture with nitrous oxide anesthesia. Anesth. Analg. 57: 283-286, 1978.

6. HiLL, D.W. Physics applied to Anaesthesia, Butterworth \& Co. (Pub.) Ltd, p. 90, 1967. 


\section{ABSTRACT}

The problems of diffusion of gas into the middle ear during anaesthesia have been described by several authors since 1965. The administration of anaesthetic concentrations of nitrous oxide raises middle ear pressure in the order of 3.43 to $3.92 \mathrm{kPa}\left(350\right.$ to $\left.400 \mathrm{mmH}_{2} \mathrm{O}\right)$. This phenomenon disappears spontaneously after cessation of nitrous oxide anaesthesia and causes no trouble except a few cases of deafness and some rare tympanic ruptures. We have tried to solve this problem during anaesthesia for tympanoplasty by substituting air for nitrous oxide and by the use of ethrane as basic anaesthetic agent. Anaesthesia was potentiated by narcotics and the patients were adequately curarized. The results showed that this technique greatly reduced diffusion of gases if we kept the $\mathrm{Pa}_{\mathrm{O}_{2}}$ under $17.9 \mathrm{kPa}(135 \mathrm{mmHg})$ and the $\mathrm{Pa}_{\mathrm{CO}_{2}}$ at $3.9 \mathrm{kPa}(30 \mathrm{mmHg})$. Controls have shown that diffusion is greater with higher $\mathrm{Pa}_{\mathrm{co}}$. Middle ear pressure, measured with Madsen impedence-meter, was not higher than $0.68 \mathrm{kPa}$ $\left(70 \mathrm{mmH}_{2} \mathrm{O}\right.$ ).

Key WORDS: ANAESTHESIA, tympanoplasty; Middle EaR, gas diffusion. 